\title{
Jorge Isaacs oder Liebe, Lesen und eine krank machende Lektüre
}

Mit dem vielberufenen Roman María von Jorge Isaacs gelangen wir zu einer Gelenkstelle zwischen circumkaribischem und andinem Raum sowie zu einer kulturellen wie literarischen Verbindung von größter Bedeutung für die Konstituierung einer relativen Einheit der verschiedenen lateinamerikanischen Literaturen. Beschäftigen wir uns also mit einem Roman, der zusammen mit José Mármols Amalia und Cirilo Villaverdes Cecilia Valdés in den lateinamerikanischen Literaturgeschichten ein romaneskes Dreigestirn bildet! Und es gibt in der Tat ästhetisch, literarisch wie literarhistorisch gute Gründe, warum María so nachhaltig kanonisiert werden konnte.

Der Roman wurde schon bald nach seiner Erstveröffentlichung $1867 \mathrm{zu}$ einem großen Erfolg und hat bis heute mehr als einhundertfünfzig spanischsprachige Auflagen erlebt. Bereits kurz nach seinem ersten Erscheinen in Kolumbien kamen in Argentinien, Mexiko und Chile Ausgaben auf den Buchmarkt, die den Erzähltext zu einem gesamtlateinamerikanischen literarischen Ereignis machten. María wurde ohne jeden Zweifel zum erfolgreichsten und meistgelesenen hispanoamerikanischen Roman des 19. Jahrhunderts.

Man dürfte wohl kaum übertreiben, wenn man María als eine Art Nationalepos Kolumbiens umschriebe und diesen Erzähltext der Reihe der novelas fundacionales im spanischsprachigen Amerika zuordnete. Genau dies ist der Grund dafür, dass wir ihn - anders als Amalia von José Mármol - in dieser der Lektüre und den Leserfiguren sowie der Liebe gewidmeten Vorlesung nur kurz und vor allem mit Blick auf die Inszenierung von Leseprozessen behandeln, obwohl es sich zweifellos um einen Text handelt, der schon für die Zeitgenossen einen geradezu kanonischen Status besaß.

Von Beginn an liegt diesem Text, der wiederum eine tragisch endende Liebesgeschichte - oder besser gesagt: deren viele - erzählt, die eingängige Grundstruktur einer Reise zugrunde. Gleich im ersten Kapitel werden wir mit einer Abschiedsszene konfrontiert; der kleine Efraín wird von seiner Familie tränenreich verabschiedet, da er sein Elternhaus verlassen muss, um nach Bogotá ins Colegio zu gehen. Dies ist ganz klar ein Standesprivileg, denn Efraín ist der Sohn eines Hacienda-Besitzers und patriarchalisch-gutdenkendaufgeklärten Sklavenhalters, der über große Besitztümer verfügt und der Elite des Landes angehört. 


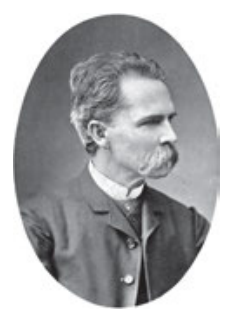

Abb. 95: Jorge Enrique Isaacs Ferrer (Cali, 1837 - Ibagué, 1895).

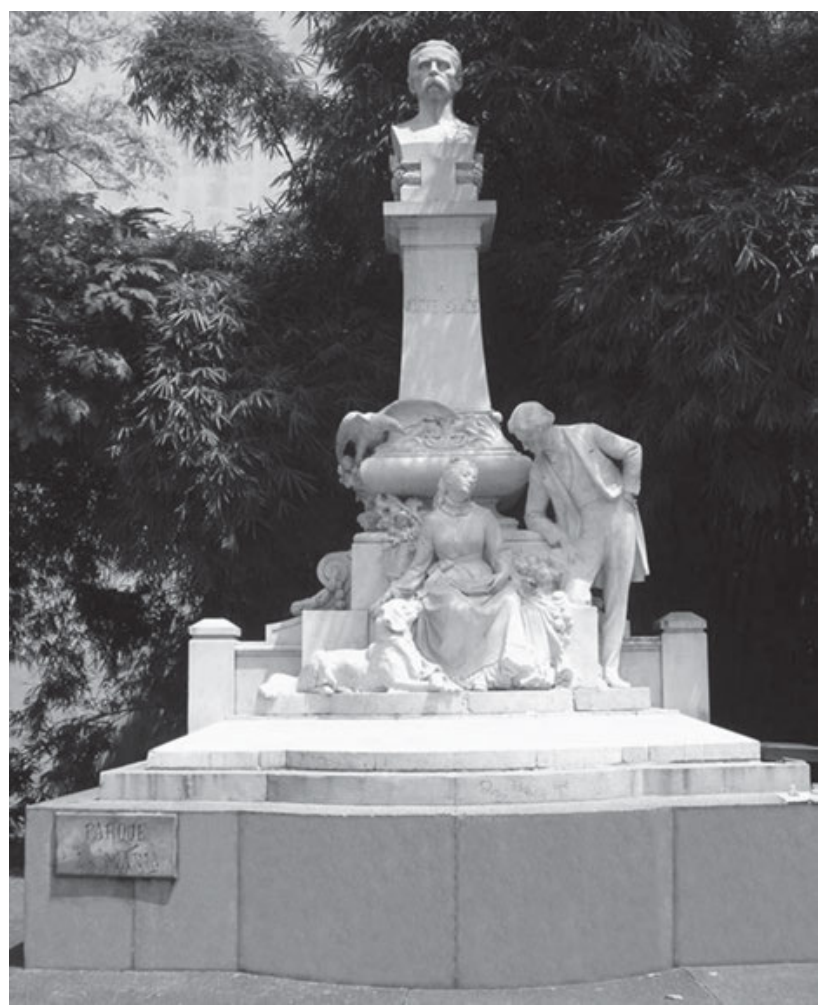

Abb. 96: Denkmal zu Ehren von Jorge Isaac mit den Hauptfiguren seines Romans María in Cali, Kolombien.

Die Abschiedsszene ist ergreifend: Mutter und Schwestern begießen den Knaben und Erben mit vielen Tränen; und ein kleines Mädchen namens María verabschiedet sich ebenfalls von ihm, nicht ohne eine erste Empfindung von Schmerz zu verspüren. Wir sehen die angehenden Liebenden im zarten Kindesalter vor uns, bevor wir sie in der Blüte ihrer Jugendlichkeit erleben werden. Freilich wird auch dann María nicht älter als fünfzehn Jahre sein. Efraín sieht sich am 
Ende des Kapitels noch einmal um und erblickt die kleine María, deren Bild wie ein basso continuo die Schlusssätze der folgenden Kapitel begleiten und mit dem der Mutter Efraíns verknüpft bleiben. Sechs Jahre später kehrt der Ich-Erzähler Efraín zurück und wieder werden die Blicke des noch hübscher gewordenen Mädchens auf ihm ruhen. Sie ist bereits zu einer jungen Frau geworden, deren kindliche Zuneigung einer entstehenden Liebe Platz gemacht hat. Efraín und María sind im Grunde schon jetzt ein Paar.

Im Verlauf der folgenden Kapitel kristallisiert sich heraus, dass María die anziehende Schönheit der Jüdinnen besitzt. Diese große Schönheit wird des Öfteren biblisch semantisiert, wobei aber auch der Verweis auf eine Jungfrau Raffaellos und damit der Hinweis auf die Portraitkunst der europäischen Renaissancemalerei nicht fehl am Platze ist. Die rein materielle Schönheit der Frau genügt nicht, sie muss durch kulturelle Tradition und Kunst zusätzlich kodiert und semantisiert werden, wie schon Roland Barthes für die „klassische“ Schreibweise des 19. Jahrhunderts herausarbeitete.

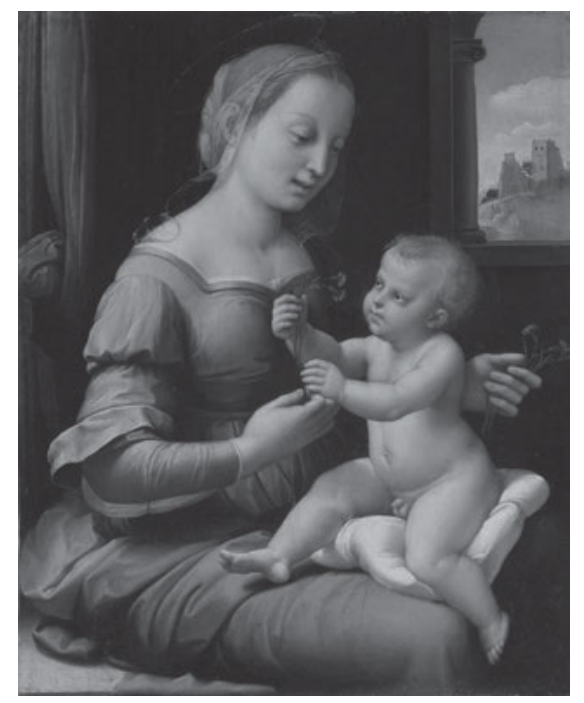

Abb. 97: „Madonna mit den Nelken“, Öl auf Eibenholz von Raffael, circa 1506-1507.

Bald schon erfahren wir, dass die Helden von Isaacs unter „nuestra raza“ die jüdische verstehen, ist doch Efraín der Sohn eines zum Christentum konvertierten Juden. Denn dessen künftige Schwiegermutter machte eine Konversion zur Vorbedingung der Hand ihrer Tochter. So verliebte sich der Vater, heiratete Efraíns Mutter aus Liebe, ließ sich in Kolumbien nieder und wurde zu einem Kolumbianer, der zur Elite des Landes aufstieg. Autobiographische Parallelen liegen auf der Hand, ist doch auch Isaacs Vater - der Familienname deutet es 
bereits an - jüdischer Abstammung und dereinst aus Jamaica nach Kolumbien eingewandert.

María ihrerseits musste sich an ihren Namen erst gewöhnen, wurde sie doch als Tochter eines jüdischen Freundes von Efraíns Vater unter dem Namen Esther geboren und erst mit drei Jahren christlich getauft. Nach dem frühen Tode ihrer Mutter Sara wurde sie von den Antillen, wohin sie deren Ehemann Salomon, der Vetter von Efraíns Vater, geführt hatte in die Obhut der Familie gebracht: ein Waisenkind also. María ist die Kusine Efraíns und hat einen zweifachen Wechsel hinter sich, nämlich den des Namens wie der Religion. Doch sie hat es nicht schlecht getroffen!

Denn ihre neue Familie ist zu Ansehen und Reichtum gekommen. Die Besitzungen im kolumbianischen Cauca-Tal werden immer größer, die paternalistisch geführten Plantagen mit ihren Sklaven scheinen guten Gewinn abzuwerfen, die Geschäfte auf Grundlage der Sklaverei blühen. Und so kann sich der Vater überlegen, seinen einzigen männlichen Nachkommen nach England und wir sehen: nicht nur unter kulturellen, sondern auch unter wirtschaftlichen und edukativen Aspekten hat London längst Madrid den Rang abgelaufen - zur Weiterbildung zu schicken. Efraín soll binnen vier Jahren sein Medizinstudium in England absolvieren und abschließen, so ward es beschlossen. Wenn da nicht die Liebe wäre! Nun schiebt sich das Motiv der Reise zwischen zwei Liebende und trennt sie, denn um ebensolche handelt es sich spätestens seit einer gemeinsamen Lektüre, die das Grundmotiv des Buchs im Buch deutlich vor Augen führt. Wir stoßen an dieser Stelle einmal mehr auf Dante Alighieris Paolo und Francesca in ihrer unübertroffenen Verknüpfung von Liebe und Lesen. Doch das Motiv erfährt bei Jorge Isaacs, wie wir noch sehen werden, eine in jeglicher Hinsicht starke Abwandlung.

Zwischen María und ihrem Cousin Efraín liegen wohl etwa vier Jahre Unterschied, so dass der Student in spe zu diesem Zeitpunkt etwa achtzehn bis neunzehn Jahre alt gewesen sein dürfte. Der junge Mann sehnt sich nach Liebe und schon tritt bei seiner Rückkehr von einem Ausflug in die Anden das Bild des Mädchens vor sein inneres Auge. Efraín befindet sich im Garten Eden, der mit allen Attributen und Ingredienzien eines locus amoenus, eines Lustortes, ausgestattet ist; doch es fehlt ihm noch die unschuldige Jungfrau, die aus der üppigen Pflanzenwelt hervortritt. Und eben diese Rolle nimmt María ein ...

María ist folglich nicht nur Esther, sondern verbindet deren jüdische Schönheit zugleich mit jener der Urmutter Eva. Sie sehen, welch geradezu archetypischen Frauenbilder sich hinter dem emblematischen Namen der Protagonistin und Titelheldin verbergen. So überrascht uns nicht, dass sie die azucena, die weiße Madonnenlilie, im Haar trägt. Auf die im Roman ständig vorgetragene 
Blumensymbolik kann ich nicht eingehen, sondern nur darauf verweisen, dass die Lilie hier für die Keuschheit Marías und zugleich die Liebesbeziehung zwischen ihr und Efraín steht. Ein wenig so, wie die Catleya für die weniger unschuldige Liebesbeziehung zwischen Swann und Odette zu Beginn von A la recherche du temps perdu eine leicht $\mathrm{zu}$ enträtselnde Chiffre darstellen sollte. Näher an Jorge Isaacs María steht freilich eine andere französische Romanfigur, nämlich die berühmte Kameliendame, La dame aux camélias, die im Romanschaffen von Alexandre Dumas fils zugleich das Thema der Krankheit einführte.

In María werden zwei Motive zusammengebracht, die wir bereits im hispanoamerikanischen Roman miteinander verbunden sahen. Nirgends aber sind sie so stark in eine literarische Tradition eingebettet wie in diesem Text, und nirgendwo sonst wird damit ein drittes, nun romantisches Motiv verbunden: das einer schleichend sich manifestierenden Krankheit.

Der in Bogotá ausgebildete Efraín fungiert gegenüber den Mädchen im Hause als eine Art Lehrer, der ihnen Geographie, aber auch die Literatur - insbesondere Frankreichs - nahebringt. Dies tut er in einer memorablen Szene, die uns nicht vergessen lassen sollte, dass dieses exklusive Bildungserlebnis darauf verweist, wie es um die Ausbildungsmöglichkeiten junger Mädchen selbst der Oberschicht in Hispanoamerika (und natürlich nicht nur dort) bestellt war.

Lieblingsautor des jungen Efraín scheint Chateaubriand zu sein, von dem Le Génie du Christianisme geradezu als Pflichtlektüre gelesen werden muss. María, die beste Schülerin Efraíns, unterliegt der Zauberkraft des französischen Schriftstellers; und sie tut dies vor allem dann, wenn die Kraft der Literatur auch noch an die Unverwechselbarkeit und Einmaligkeit der ach so verführerischen Stimme des jungen Lehrers geknüpft ist, die nichts anderes darstellt als die Präsenz des geliebten Körper-Leibes.

Die gemeinsame, laute Lektüre entfaltet die volle Wucht der Verführung durch Sprache, durch ausgesprochene, verkörperte Sprache. Doch hören wir selbst! Der Ich-Erzähler Efraín liest seiner Schwester Emma und der jungen María vor, im Freien, mit Blick auf das majestätische Cauca-Tal, im Setting eines Naturgemäldes, wie es eindrücklicher (und zugleich romantischer) nicht sein kann. Dabei hat er Chateaubriands Atala gewählt, das - wie er erklärt - alle Melancholie des französischen Dichters aufgesammelt und in sich vereint habe. Wir haben es im Folgenden wieder mit einem Sonnenuntergang zu tun, diesmal aber nicht bei blanker Imagination, sondern einen Text vor Augen und zugleich in den Ohren: Chateaubriands Atala ist zurück in der Neuen Welt! Es ist wieder Nachmittag, die Lesestunde also:

An einem Nachmittage war es, einem jener Nachmittage meines Landes, die verziert sind mit Wolken von violetter Farbe und einem blassgoldenen Aufleuchten, so schön wie María, 
so schön und vorübergehend, wie sie für mich war. Da saßen María, meine Schwester und ich auf dem weiten Felsen eines Abhanges, von wo aus wir zu unserer Rechten die tiefen bebauten Talbereiche mit der schäumenden Strömung des Flusses sich schlängeln sahen, zu unseren Füßen das majestätische und stumme Tal, und ich las die Episode von Atala. Die beiden, bewundernswert in ihrer Unbeweglichkeit und Hingabe, hörten von meinen Lippen all jene Melancholie erblühen, die von diesem Dichter versammelt wurde, „um die Welt weinen zu lassen“. Meine Schwester, ihren rechten Arm auf einen meiner Arme gestützt, ihr Haupt fast mit dem meinigen vereint, verfolgte mit den Augen die Zeilen, die ich Satz für Satz las. Währenddessen wandte María, die nahe bei mir kniete, ihre schon feuchten Blicke nicht von meinem Antlitz ab.

Die Sonne hatte sich bereits versteckt, als ich mit veränderter Stimme die letzten Seiten des Gedichtes las. Emmas bleiches Antlitz ruhte auf meiner Schulter. María verbarg sich das Gesicht mit beiden Händen. Nachdem ich Chactas’ aufwühlenden Abschied über dem Grabmal seiner Geliebten gelesen, ein Abschied, der so viele Male einen Schluchzer meiner Brust entriss, „Schlafe in Frieden in fremder Erde, Du Junge und Unglückliche! Als Dank, als Vergeltung Deiner Liebe, Deiner Exilierung und Deines Todes wirst Du nun selbst noch von Chactas verlassen." María vernahm meine Stimme nicht mehr und enthüllte ihr Gesicht, über welches dicke Tränen rannen. Sie war so schön wie die Schöpfung des Dichters, und ich liebte sie mit der Liebe, welche er imaginierte. Wir schritten stumm und still langsam zum Haus zurück. Ach, meine Seele und die Seele von María waren nicht nur tief bewegt von jener Lektüre, sie waren vielmehr bedrückt von einem Vorgefühl! ${ }^{1}$

In geradezu archetypischer Weise wird in diesen Zeilen eine Aneignungsform von Literatur vorgeführt, wie sie im Grunde schon seit dem 18. Jahrhundert gefordert und gefördert wurde. Es ist eine Lektüre als Seelenverschmelzung, als tränenreiche Identifikation, die einen Idealtyp romantischen Lesens darstellt. Diese Lektüre und diese Lesart sind in den in Anführungszeichen gesetzten und übersetzten Worten des französischen Dichters Chateaubriand gleichsam als Vermächtnis dem Text Isaacs wie der Lektüresituation des dreizehnten Kapitels eingeschrieben. Chateaubriand wird noch einmal im Munde des Ich-Erzählers, aber auch in der Schrift des kolumbianischen Autors auf zweifache Weise lebendig: Atala kehrt in die Neue Welt zurück und präfiguriert das tragische Schicksal der Liebenden, über denen eine böse Vorahnung schwebt.

Zugleich wiederholt sich der Aufbau der Lesesituation, insoweit hier eine männliche Stimme aus einem Text der (Früh-)Romantik vorliest und die Frauen sich angesichts dieser lauten Lektüre ganz in die Magie des Textes und des männlichen Vor-Lesers begeben. Die Nähe zum Inzest wird durch die Nähe der Schwester dargestellt, die sich auf den Arm - der sicherlich psychoanalytisch

1 Isaacs, Jorge: María. Prólogo de Eduardo López Morales. La Habana: Casa de las Américas 1975, S. 33. 
leicht deutbar wäre - ihres Bruders stützt und damit jenes weibliche Begehren verkörpert, das die schon feuchten Augen Marías zum Ausdruck zu bringen versuchen. So also wird der in der Neuen Welt angesiedelte tränenreiche Roman Chateaubriands, der erstmals 1801 von Fray Servando Teresa de Mier ins Spanische übersetzt wurde und einen beispiellosen Siegeszug gewiss nicht nur in Amerika antrat, im Süden des Neuen Kontinents, im kolumbianischen Cauca-Tal, produktiv rezipiert.

In diesem Zusammenhang wird deutlich, was Jean-Jacques Rousseau meinte, als er von der dangereuse méthode einer gemeinsamen (und überdies lauten) Lektüre sprach. Denn der lesend angeeignete französische Roman nimmt alle Gefühle der Protagonisten vorweg, noch bevor diese selbst gefühlt werden können. Man könnte sich mit guten Gründen sogar fragen, ob sie ohne ihn, ob sie ohne die Lektüre überhaupt gefühlt werden könnten. Denn alles hängst von den Seiten des Buches und zugleich von den Lippen des Vorlesers ab; diese Seiten und mit ihnen die tragische Liebe werden genau so angeeignet, wie der Dichter dies zu Protokoll gegeben und buchstäblich imaginiert hatte.

Daher kommt Chateaubriands Atala auch eine schicksalhafte Dimension zu: Denn in dieser Passage wird gleichsam das Schicksal der beiden Liebenden präfiguriert, also in einer Art und Weise vorweggenommen, die die Literatur zur Wirklichkeit, das Lesen zum wahren Leben macht. Kein Wunder also, wenn María nach der gemeinsamen Lektüre in ihrer Gesundheit stark angegriffen ist und krank wird. Es waren der Aufregungen zu viele. Das bildschöne Kind, das nicht umsonst in das Umfeld der schönen Atala gestellt wird, verwandelt sich in ein Opfer der gemeinsamen Lektüre.

Dies ist die eigentliche Schlüsselszene des gesamten Romans, in ihr bündeln sich intertextuell verankert alle Isotopien. Die Situation der gemeinsamen lauten Lektüre ist dabei ein Element literarischer Tradition, die an das Schicksal von Paolo und Francesca im fünften Gesang des Inferno in Dantes Göttlicher Commedia erinnert: „An jenem Tage lasen sie nicht weiter“. Natürlich ist im Blick vom Berge in die weite Fläche auch ein Stückchen Petrarca enthalten, insofern sein Aufstieg zum Mont Ventoux einstmals von der Lektüre im Buch gekrönt worden war und eine Schlüsselszene in der Geschichte der Konstituierung moderner Subjektivität darstellt. ${ }^{2}$

Die gesamte Szenerie ist also literarisch beziehungsweise literarhistorisch vorbestimmt, wird aber bereits situativ mit der Sonnenuntergangsszene romantisch aufgeladen. Auf einer zweiten Ebene nun wird der Intertext, Chateaubriands

2 Vgl. hierzu Ritter, Joachim: Landschaft. Zur Funktion des Ästhetischen in der modernen Gesellschaft. In (ders.): Subjektivität. Sieben Aufsätze. Frankfurt am Main: Suhrkamp 1989, S. 141-163. 
Atala, in den Text von Jorge Isaacs als ins Spanische übersetztes Zitat eingebaut, wird also zu einem integralen Bestandteil des eigenen Textes, wenn auch explizit mit Gänsefüßchen versehen. Wir haben einen Übersetzungsvorgang in mehrfacher Hinsicht vor uns, der sich nicht nur auf der sprachlichen und kulturellen, sondern auch auf einer lebenspraktischen (und lebenswissenschaftlich aufschlussreichen) Ebene ansiedelt. Damit ist nicht nur die Tatsache gemeint, dass Atala ins Spanische übersetzt ist, im Übrigen realhistorisch auch insoweit interessant, als Atala just in Kolumbien von einem der großen kolumbianischen Schriftsteller ins Spanische übersetzt worden war. Denn Atala wird qua Lektüre zum Paradigma des eigenen Lebens von Isaacs Gestalten: María wird so schön wie die Schöpfung Chateaubriands und der Ich-Erzähler selbst liebt diese Gestalt Chateaubriands mit eben jener Liebe, die der französische Autor seinen Gestalten einflößte. Efraín verwandelt sich folglich zu einem zweiten Chactas.

Damit ist bereits der weitere Lebens- und Sterbensweg der schönen AtalaMaría vorgezeichnet, die unser Chactas-Efraín am Ende des Romans besuchen wird. Eine wahrhaft körperlich-leibhaftige Anverwandlung eines Intertexts! Die gelesene Literatur wird zur lebenspraktischen Vorgabe romanesker literarischer Gestalten: Jorge Isaacs zeigt auf, wohin die Lektüre führen kann und warnt zugleich vor den Folgen anverwandelter Literatur. Sein männlicher Protagonist Efraín bleibt diesem Modell treu, wird er doch nicht nur in seiner Jugend die verstorbene Geliebte beweinen, sondern auch noch als Ich-Erzähler am Ende seines Lebens und am Ende des Romans das Grabmal Marías aufsuchen und ihr Schicksal untröstlich betrauern. Damit ist zugleich eine Lektürevorgabe gemacht und ein Warnschild eingeblendet.

In diesen Passagen geschieht allerdings im Grunde nichts anderes als das, was wir schon in mehreren Romanen der Subgattung novela sentimental hatten beobachten können: die kreative Integration vor allem von Texten der französischen Romantik. Dabei bricht Chateaubriands Atala ohne jeden Zweifel alle Rekorde der Zitierhäufigkeit. Der französische Bezugstext stellt so etwas wie einen Urmythos dar und die hispanoamerikanischen Romane leisten dabei (ganz im Blumenberg'schen Sinne) ihre Arbeit am Mythos. Der exotisierend nach Amerika verlagerte Text des Franzosen wird in Amerika reexotisierend gelesen und auf Ebene der Autoren in eigene literarische Praxis, auf Ebene ihrer Gestalten in konkrete Lebenspraxis verwandelt. Jorge Isaacs María erzielte dabei den größten Lese- und wohl auch Verkaufserfolg.

Von besonderer Bedeutung scheint mir dabei die Tatsache zu sein - und sie ist nicht in das Paolo-und-Francesca-Motiv Dantes aufzulösen -, dass hier die Stimme des geliebten Wesens an (oder besser in) das Ohr der geliebten Frau eindringt. Stimme und Körper stehen im Roman für das Unverwechselbare, für 
das vom Körper garantierte Authentische ein. Auf diese Weise wird gerade das Zitat durch den Prozess kreativer körperlicher Anverwandlung zum eigenen Klangtext, hier zum authentisch empfundenen Beleg des eigenen Gefühls, das die Seelen durchdringt, die Körper erschüttert und den schönen Leib der jungen María langsam, aber sicher ruiniert: Killing me softly with his song.

So wird auch verständlich, warum María ihre Augen nicht mehr zur Informationsaufnahme, sondern nur noch zur Gefühlsausgabe verwenden kann: María weint und weint ... Und sie wird es im Roman noch so oft tun, dass ein Kritiker einmal schreiben zu müssen glaubte, dass sie nicht an einem epileptischen Anfall, sondern an Wasserentzug gestorben sei. Die vollständige Identifikation, dies zeigt auch der Kommentar des Ich-Erzählers, vollzieht sich zwischen dem ursprünglichen Autor und dem Vorlesenden sowohl aus der Perspektive des Lesenden als auch aus jener der Hörenden. Die Aneignung des Textes durch die Stimme, durch den Körper, verwandelt den Text in etwas, das aus dem Körper des Vortragenden selbst kommt und diesen re-präsentiert. Die Reaktion der beiden Frauen ist - um das obige übersetzte Zitat noch einmal im Original vorzuführen deutlich: „Las dos, admirables en su inmovilidad y abandono, oían brotar de mis labios toda aquella melancolía aglomerada por el poeta para ,hacer llorar al mundo“. "His Master’s Voice: Doch hinter der Stimme des Herrn verbirgt sich eine andere Stimme des Herrn, für welche die Stimme des liebenden Subjekts nicht mehr als die Stimme eines Bauchredners ist. Roland Barthes hat in seinen Fragments d'un discours amoureux diese Kon-Figuration zur Genüge erläutert.

Die Worte kommen einem natürlichen Prozesse folgend gleichsam sprießend von den Lippen des Lesenden: María wird Efraín gegenüber im vielfachen Sinne hörig. Das fremde Wort kommt aus dem Inneren des männlichen Körpers. Allein in der Schriftlichkeit wird es mit Hilfe der Anführungszeichen noch als fremdes Wort erkennbar und markiert. Als angeeignetes Zitat aber ist es für die beiden Mädchen nicht mehr erkennbar, sie geben sich diesen Worten unbeweglich hin. Die Worte dringen in ihre Ohren, in ihre Körper ein. Die Lust am Text, jenes erotische Moment der lauten Lektüre, der lecture à haute voix, von der ebenfalls Roland Barthes am Ende von Le Plaisir du texte sprach, wird hier in ihrer Wirkungsweise vorgeführt.

Die Verinnerlichung des fremden Wortes führt auch zur Verinnerlichung des fremden Lebens: María und Efraín folgen ihren Vorbildern Chactas und Atala bis in Details. Es wäre ein Leichtes, dies an einer Vielzahl von Passagen in Jorge Isaacs Text zu belegen. Und gerade hier setzt auch die Krankheit Marías an, die wie bei Atala von innen kommt. Sie ist weniger ein Erbgut ihrer früh verstorbenen Mutter Sara als ein Erbgut der französischen Romantik. Lektüre, so dürfen wir folgern, kann krank machen. 
Denn gleich im folgenden Kapitel erleidet sie ihren ersten Kollaps, eine Art epileptischen Anfall. Wer die Bedeutung dieser Szene noch nicht verstanden hat, wird sogleich von Autor und Erzähler durch Gewitter und starke Regenfälle daran erinnert, wie eng alles in Isaacs' Roman mit Atala verzahnt ist und wie sehr die Natur gerade im hispanoamerikanischen Roman einen aktiven Part übernimmt. Klar ist auch, dass Marías Krankheit unheilbar ist, wie ein herbeigezogener Arzt mit dem notwendigen wissenschaftlichen Aplomb verkündet. Der Mann war leider Schulmediziner! Eine Literaturwissenschaftlerin oder ein Literaturwissenschaftler hätten nicht nur eine Diagnose, sondern auch eine Therapie gefunden. Sie hätte wohl ,kritische Lektüre‘ geheißen. So aber erfolgt allenfalls der Ratschlag, dass man María jegliche Art von Emotionen ersparen müsse. Efraín wird sogleich von seinem Vater darauf hingewiesen, dass er als künftiges Familienoberhaupt Verpflichtungen für die ganze Familie trage: an eine Heirat mit María sei nicht zu denken! Genau daran aber denken die Liebenden, die nicht mehr wissen, wie sie sich nun verhalten sollen.

Lesen und Lektüre stehen nicht nur inhaltlich, sondern auch strukturell und semantisch ganz im Zentrum dieses Romans, den man mit Vorbehalten durchaus als einen Leseroman bezeichnen kann, welcher um eine heterosexuelle Liebesbeziehung kreist. Er schwankt - mit der argentinischen Literaturwissenschaftlerin Susana Zanetti gesprochen - zwischen einer archaischen und einer modernen Art der Aneignung oder ebensolcher Lektüremodi. ${ }^{3}$

Erst im vierunddreißigsten Kapitel des Romans werden sich die Liebenden wiedersehen; und zum ersten Mal erblickt der Ich-Erzähler Efraín nach seiner Rückkehr aus Übersee María just dort, wo er sie bei der gemeinsamen Lektüre krank machend in seinen Bann gezogen hatte: auf einem jener großen Felsen, die das Cauca-Tal überragen. María ist kaum zu bewegen, von diesen Felsen herabzusteigen, so sehr hat sich das Schicksal Atalas mitsamt der Melancholie, dem mal de siècle, auf ihre Stimmung und ihr ganzes Leben gelegt. Nur schwer kommen die beiden Liebenden wieder in ein Gespräch, das damit beginnt, dass Efraín María danach fragt, was sie während seiner langen Abwesenheit denn getan habe. María weiß zunächst nicht, was sie antworten soll, bis die Frage auf die Liebe und das Lesen kommt:

3 Vgl. Zanetti, Susana: La dorada garra de la lectura. Lecturas y lectores de novela en América Latina. Rosario: Beatriz Viterbo 2002. 
- Was hast Du während dieser Tage getan?

- Gewünscht, dass sie vorübergingen.

- Nichts weiter?

- Nähen und viel denken.

- Woran?

- An viele Dinge, die man denkt, aber nicht ausspricht.

- Nicht einmal an mich hast Du gedacht?

- An Dich am wenigsten.

- Ja, gut.

- Denn Du weißt es ja.

- Hast Du nicht gelesen?

- Nein, denn alleine zu lesen macht mich traurig, und mir gefallen nicht länger die Veladas de la Quinta oder die Tardes de la Granja. Ich wollte Atala wieder lesen, aber da Du gesagt hast, dass es da eine Stelle gibt, ich weiß nicht wie ...

[...]

Wir öffneten die Türe und sahen auf einem der Fensterflügel, welche der Wind hin- und her bewegte, einen schwarzen Vogel von der Größe einer sehr großen Taube sitzen: Er gab einen Schrei von sich, wie ich ihn noch nie gehört habe; er schien einen Augenblick lang durch das Licht, das ich in meiner Hand hielt, glühende Augen zu bekommen, und er löschte es aus, als er über unsere Köpfe hinwegflog, während wir erschrocken die Flucht ergriffen. In jener Nacht träumte ich ... Aber was ist mit Dir? ${ }^{4}$

In dieser Passage wird deutlich und schön vorgeführt, wie sehr die Lektüre den Schlüssel zum gesamten Text enthält. Denn das Denken und Fühlen ist immer an das Lesen gekoppelt, genauer noch: an die gemeinsame Lektüre, welche María nicht mehr vergessen kann. Denn zwei Arten von Lektüren fallen nunmehr weg: die Lektüre ebenfalls französischer, aber letztlich wenig aussagekräftiger, harmloser da noch nicht romantischer Texte (die Susana Zanetti näher besprochen hat, ${ }^{5}$ die uns hier aber nicht näher interessieren), Texte also, die nichts von der Wirkung der Texte Chateaubriands besitzen. Atala wiederum kann aber nur in gemeinsamer, gleichsam archaischer Lektüre angeeignet werden, nämlich mit Hilfe einer vereinten Lektüre beider Liebender. Doch längst ist ein schwarzer Vogel über die Seele der jungen Frau hinweggeflogen.

Die einsame Lektüre ist für María keine Alternative, keine praktikable Lösung, noch nicht einmal ein guter Zeitvertreib. Sie vermittelt der verliebten Frau das Gefühl, völlig alleine zu sein; außerdem gibt es da ja auch noch so eine Stelle, eine Passage, vor der Efraín sie gewarnt hatte - wobei das je ne sais quoi, das stets die irrationalen Restbestände anzeigt, hier durchaus zwischen Eros und Thanatos zu verstehen und im Bereich des Irrationalen anzusiedeln ist. Längst ist Efraín die

4 Isaacs, Jorge: María, S. 159.

5 Vgl. Zanetti, Susana: La dorada garra de la lectura, S. 185-205. 
Gefährlichkeit der Lektüre deutlich geworden: Sein Vorlesen war es ja, das María so tief ins Innere traf. Ist er sich seiner Mitschuld bewusst?

Dann aber schlägt - ganz wie bei José Mármol - das Lesen des Textes um in ein Lesen der Zeichen der Natur, und auch diese sind nicht weniger fatal und furchterweckend wie in Mármols Roman Amalia. Denn der schwarze Vogel, gleichsam Edgar Allan Poes The Raven, krächzt erheblich durch die Seiten, so dass der Ich-Erzähler auch erschrickt; denn er weiß früher als die nur im Traum reagierende María die bösen Vorzeichen zu deuten. Die Lesbarkeit der Welt ist ausgerichtet an einer Leseschule, die freilich furchtbare Konsequenzen für die Gesundheit der Leser und insbesondere der Leserinnen besitzt. Denn auch die Lektüre der Zeichen der Natur kann krank machen.

Wir verkürzen die Fabel des Romans ein wenig, indem wir lediglich darauf aufmerksam machen, dass erneut das Motiv der Reise nach Europa - wie bei Enrique Otway in Gertrudis Gómez de Avellanedas Sab zur Ausbildung in England - Trennung und Tod herbeiführen beziehungsweise beschleunigen. Wir wollen uns nicht mit der Vielzahl in den Romanablauf integrierter kostumbristischer Szenen oder mit den eingebauten zusätzlichen Liebesgeschichten auseinandersetzen, die nicht nur der Zerstreuung und Spannungserzeugung bezüglich der zentralen Liebesbeziehung dienen, sondern auch eine Vielzahl verschiedener Kultur- und Lebensformen in die Romandiegese einblenden, die soziale Spannbreite des Romans erhöhen und zugleich in unterschiedlichen diegetischen, gesellschaftlichen und kulturellen Kontexten die immer gleiche Liebesgeschichte durchexerzieren!

Die Liebe proliferiert als narrativer Treibsatz so sehr, dass sich ständig Doubletten bilden, die als retardierende Momente gegenüber der Hauptliebesgeschichte auf diese strukturell bezogen sind. Die Sklavenjagd in Afrika, deren Vertreter und Opfer, die Bewohner der kolumbianischen Karibikküste, die Siedler im Caucatal, die Bauern hoch oben in den Anden: Sie alle werden mit ihrer jeweilig stilisierten Sprache eingeblendet, wobei es nicht an Missverständnissen, Quiproquos und komischen Effekten mangelt. Eine Tigerjagd oder die gefährliche Durchquerung eines reißenden Flusses auf dem Pferd, die Flussfahrt auf dem Río Magdalena frei nach Alexander von Humboldt (dessen Bericht zweifellos tiefe Spuren hinterlassen hat) und die Streifzüge durch die Anden: All dies entfaltet ein Panorama, das man sehr wohl mit einem Begriff des Schöpfers der Ansichten der Natur als ein literarisches Naturgemälde bezeichnen darf. Es gibt auch in María eine Einengung des Blickes und eine Konzentration auf die Geschehnisse rund um die Titelheldin, doch wird eine derartige Verengung mit Hilfe des Kunstgriffs zusätzlicher Liebesgeschichten überwunden oder zumindest konterkariert. 
Während der Aktionsradius unseres Tigertöters Efraín schon vor seiner Abreise nach England immer größer wird, schrumpft die Welt der fünfzehnjährigen María immer weiter. Ihre Schönheit ist mehr und mehr die einer romantischen bellezza: Sie ist schon jetzt bleich, ja totenbleich. María wird sukzessive zu jener romantischen Frauenleiche, über welche die Geschichten, welche uns der hispanoamerikanische Roman der Romantik zu erzählen hat, fast zwanghaft und obsessiv verlaufen. Auch hier gilt Elisabeth Bronfens Diktum mit aller Schärfe: Nur über ihre (schöne) Leiche. ${ }^{6}$

Carlos, ein ehemaliger Schulfreund von Efraín, war gekommen, um seinerseits um die Hand der bildhübschen María anzuhalten. Doch rasch erkennt er die starke Liebe seines Freundes und fragt ihn im achtundzwanzigsten Kapitel des Romans nachdrücklich angesichts der Krankheit des Mädchens, ob dieser denn ein halbes Leben lang auf dem Grabstein Marías sitzen wolle. Wir könnten ihm an dieser Stelle die philologische Antwort geben, welche die Lektüreszene uns bereits vorgab: Efraín verhält sich ganz wie Chateaubriands edler Indianer Chactas. Doch der nüchternere Carlos, der sich über die Lektüren Efraíns genüsslich lustig macht, hat erkannt, worum es hier geht: um die mehr oder minder kreative Anverwandlung romantischer Liebes- und Lebensmuster. Efraíns Freund hat bald verstanden, welche die Quellen aller Übel sind: die vermaledeiten Bücher! Schauen wir also einmal mit Carlos in Efraíns Bibliothek, in das Innerste eines jungen gebildeten Lesers im kolumbianischen 19. Jahrhundert, um etwas mehr über die spezifischen Aneignungsmuster in dieser Bücherexaminierungsszene zu erfahren:

- Schauen wir mal!, und er begann die Buchrücken zu lesen, Frayssinous, Christus vor dem Jahrhundert, Die Bibel ... Hier ist viel Mystisches. Don Quijote ... Na klar doch: Ich selbst habe nicht einmal zwei Kapitel davon lesen können.

- Nein wirklich?

- Blair, machte er weiter, Chateaubriand ... Meine Kusine Hortense ist ganz verrückt danach. Grammatik der englischen Sprache ... Was für eine rebellische Sprache! Da fand ich nie rein.

[...]

- Shakespeare? Calderón ... Verse, nicht wahr? Spanisches Theater. Noch mehr Verse? Komm', gestehe es mir: Schreibst Du noch immer Verse? Ich entsinne mich, dass Du welche machtest, die mich traurig stimmten, wenn ich an den Cauca dachte. Also machst du noch immer welche?

- Nein.

- Da bin ich aber froh, denn sonst würdest du am Ende noch an Hunger sterben.

- Cortés, machte er weiter - die Eroberung Mexikos?

6 Vgl. Bronfen, Elisabeth: Nur über ihre Leiche. Tod, Weiblichkeit und Ästhetik. Würzburg: Königshausen u. Neumann 1994. 
- Nein; das ist etwas anderes.

- Tocqueville, Über die Demokratie in Amerika ... Die Pest! Segur ... Wie Langatmig!

Bis dahin kam er, als das Glöckchen des Speisesaales erklang und ankündigte, dass eine Erfrischung serviert war. Carlos hörte mit seiner Fiskalisierung meiner Bücher auf, näherte sich dem Spiegel und kämmte sich seinen Backenbart und die Haare mit einem Taschenkamm, faltete seine blaue Krawatte wie eine Damenschneiderin ihr Band, und wir gingen hinaus. ${ }^{7}$

Wir haben es hier mit einer ,klassischen` Bücherexaminierungsszene und damit dem Phänomen des Buches im Buch zu tun. Diese Szenerie aus dem zweiundzwanzigsten Kapitel des Romans ist wunderschön ironisch angelegt, gibt zugleich aber den Blick frei auf jene Denkmuster und Aneignungsformen, die sozusagen in der Bibliothek des ehemaligen Dichters Efraín gespeichert sind.

Der anfängliche Verweis auf Frayssinous' Défense du christianisme von 1825 - wie zuvor auf Chateaubriands Le Génie du Christianisme - verweist im Kontext anderer erwähnter Werke auf den christlichen und sicherlich auch religiös-mystisch-devoten Hintergrund der Bildung des jungen Kolumbianers, Blair wiederum auf die Lecciones sobre la Retórica y las Bellas Artes und damit auf den künstlerisch-rhetorischen Hintergrund der literarischen Lebensaneignung Efraíns; auch dies eine Komponente einer geschickten literarischen Konstruktion, in der wir leben, was wir lesen. In der Bücherexaminierungsszene ist folglich eine ganze Ästhetik des Romans versteckt.

Der Hinweis darauf, dass die Kusine Hortense ganz wild auf Chateaubriand sei, ruft bestimmte Aneignungsmuster auf, die wir schon kennen, die für Carlos aber nicht positiver Natur sind. Die Kontrolle und Pflege der Sprache wiederum interessiert ihn überhaupt nicht und mit Grammatiken hat er nicht viel im Sinn. Shakespeare und Calderón stehen ganz ohne Zweifel für die von den Romantikern aufgewerteten ,Klassiker ' der englischen und spanischen Literatur des 16. und 17. Jahrhunderts, gleichsam eine literarische Basis, auf welche die neueren Dichter zurückgreifen können. Eine europäische Literaturgeschichte in nuce entsteht, freilich am Beispiel einer in Amerika angesiedelten Bibliothek. Wir sehen, wie stark der Pol abendländischer Kultur- und Literaturtraditionen ins Gewicht fällt.

Der anschließende Hinweis auf Cortés meint nicht den berühmten Eroberer Mexicos, sondern einen anderen gleichnamigen Autor des Buches Ensayo sobre el catolicismo, el liberalismo y el socialismo von 1851, also ein Hinweis auf den liberalen politischen Hintergrund Efraíns, der auch durch die Auffindung von Alexis de Tocquevilles De la Démocratie en Amérique verstärkt wird.

7 Isaacs, Jorge: María, S. 96f. 
Doch bevor die Examinierung oder - wie es im Text heißt - Fiskalisierung mit Madame de Ségur weitergehen kann, werden beide unterbrochen. Für Carlos ist der Blick in den Spiegel keine Selbsterkenntnis, keine Veränderung und Bewusstwerdung des eigenen Lebens, sondern dient nur der optischen Oberflächenkontrolle, die sich weit jenseits der Dinge ansiedelt, welche Efraín so umtreiben.

Wir aber wissen nun, wes Geistes Kind Efraín ist, kennen die Gedankenmodelle, die seinen Geist geprägt haben und wohl auch noch immer prägen. So erfüllt die Bibliothek im Buch den Zweck, eine bestimmte Person zu situieren, ihren gebildeten Status zu untermauern und den Raum als Teil eines Hauses zur Geltung zu bringen.

Aufschlussreich ist, dass die Romanfiguren verschiedentlichen Namenswechseln unterliegen. María hieß ursprünglich Esther, die Sklavin Feliciana Nay, und auch andere Figuren haben ihre Namen gewechselt oder andere Namen gewählt. Erneut ist das Problem von Fremdbestimmung und Namensgebung vorhanden, wie es dem gesamten Kontinent und seiner Namensgebung zugrunde liegt. Die Namenswechsel verweisen auch auf Transkulturationsvorgänge: Spanier, Kreolen, Juden, Schwarze, Mulatten, Briten und viele mehr sind die Bewohner dieses kolumbianischen Nationalepos, das auch die Koppelung ethnischer mit sozialen Grenzen belegt. Die Namenswechsel sind Zeichen einer Transkulturation, die sich in den heterogenen Populationen des Kontinents zeigt.

Der Tag der Abreise Efraíns nach England rückt näher. Die Lippen der geliebten Frau bleiben geschlossen, die Blicke der Verängstigen sprechend dafür aber eine umso deutlichere Sprache. Die Briefe, die sie nach England schickt, und mehr noch deren Ausbleiben, künden von der fortschreitenden Krankheit, die sich immer mehr verstärkt und der sie schließlich erliegen wird. Die Liebenden werden sich im Leben nicht wiedersehen! Dem übereilt nach Kolumbien zurückkehrenden Efraín bleibt nur jene Position, die ihm Chateaubriands Atala bereits zuwies: die des Liebenden am Grabe seiner Geliebten, untröstlich weinend und ganz der Melancholie verfallen.

$\mathrm{Zu}$ den literarischen Höhepunkten des Romans zählt zweifellos die ausführlich dargestellte Flussfahrt den Río Magdalena beziehungsweise den Río Cauca hinauf. Kostumbristische Szenen mischen sich mit naturgeschichtlichen Erläuterungen der Erzählerinstanz: Nicht umsonst wurde der Roman von Jorge Isaacs als kolumbianisches Nationalepos gelesen. Majestätisch-traurig sind die Nächte am Cauca; sie zögern nur die Enttäuschung (und Entladung der Spannung) des aus England heimkehrenden Efraín hinaus. Die an Alexander von Humboldt geschulten Beschreibungen einer großartigen Natur - auch Humboldt war ein 
aufmerksamer Leser Chateaubriands, dem er bisweilen laut aus seinen Schriften vortrug $^{8}$ - besitzen nicht nur eine verzögernde Funktion innerhalb des Plots im Sinne eines suspense, sondern unterstreichen zugleich die Symbolik des Wassers, die diesen kolumbianischen Roman prägt.

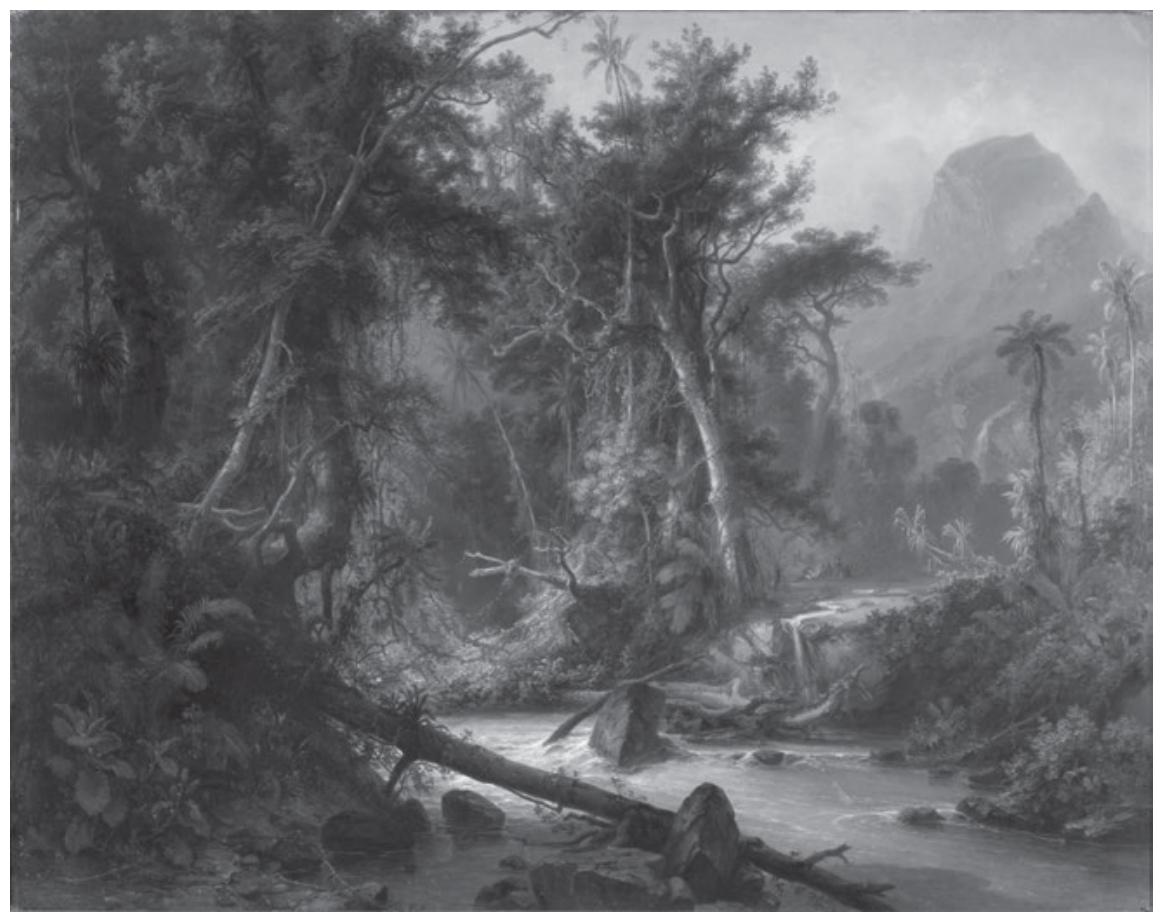

Abb. 98: „Südamerikanische Urwaldlandschaft mit Ureinwohnern am Lagerfeuer“, Öl auf Leinwand von Ferdinand Bellermann, 1863.

Efraíns Reisetagebuch ist noch voller Hoffnung auf das Künftige, auf die Verwirklichung seiner Liebesgeschichte. Doch seine Flussreise ist die Reise zum Tod der Geliebten, eine Reise ähnlich jener entlang der Ufer des Mississippi, wie sie bei Chateaubriand erscheint. Schon beim ersten Anfall Marías war der Fluss, den Efraín auf seinem Weg zum Arzt durchqueren musste, beträchtlich angeschwollen. Im Romanverlauf deutet dieses Anschwellen, „la creciente“, mehrfach auf

$8 \mathrm{Zu}$ den Beziehungen des Preußen zur französischen Literatur und ihren Schriftstellern vgl. Lenz, Markus Alexander: Französische Literaten. In: Ette, Ottmar (Hg.): Alexander von Humboldt Handbuch. Leben - Werk - Wirkung. Mit 52 Abbildungen. Stuttgart: Metzler 2018, S. 229-235. 
das Sterben und den Tod. ${ }^{9}$ Ein „torrente“, ein Sturzbach durchläuft jenen Garten, in welchem sich María am Ende aufhält; Wasser bildet die Straße, die Efraín zu ihrem Grab führen wird; und Wasser waren auch die Tränen des Abschieds, welche vom ersten Kapitel an den Roman und seine Titelheldin begleiteten - bis hin zu jenem Felsen hoch über dem Cauca-Tal, wo sich bei der fatalen Lektüre die Strömung des Flusses hinter den Lesenden abzeichnete und ihre Windungen tief in die großartige Naturszenerie einschrieb.

Das Unausweichliche vollzieht sich: María stirbt und verlässt diese Erde, einem Engel gleich... Dem Ich-Erzähler bleibt nur das Aufsuchen der mystischen Orte ihrer Vergangenheit zu zweit, darunter auch jener der gemeinsamen Lektüre hoch oben auf dem Felsen. ${ }^{10}$ Der Roman endet an einem Ort, der ihm früh schon von Chateaubriands Atala vorgegeben worden war: am Grabmal der schönen María. So lesen wir auf der letzten Seite:

Die untergehende Sonne durchkreuzte das verzweigte Blattwerk des nahe gelegenen Tropenwaldes mit einigen ihrer Strahlen und ließ die Brombeergebüsche und die Blätter der Bäume, welche den Gräbern Schatten spendeten, gelb aufleuchten. Als ich einer Gruppe korpulenter Tamarinden meinen Rücken zuwandte, stand ich vor einem weißen und von den Regenfällen befleckten Sockel, über dem sich ein eisernes Kreuz erhob: Ich trat näher. Auf einer schwarzen Tafel, welche die Zweige der Frauenpantöffelchen halb verdeckten, begann ich zu lesen: „María“..."

Am Ende steht ein letzter Lesevorgang, eine letzte Lektüre. Und wieder gilt diese Lektüre der Liebe, einer tragisch zu Ende gegangenen Liebe. Wir haben im Verlauf unserer Vorlesung bereits eine ganze Menge solcher Kreuze gesehen, die den Text mit einem ,Ruhe in Frieden“ inmitten einer Friedhofsarchitektur abschließen. Ich erinnere hier nur an das Kreuz in Gertrudis Gómez de Avellanedas Sab oder eben in Chateaubriands Atala.

In Jorge Isaacs Narrativ hat der Roman mit diesen Sätzen seinen intertextuell vorgezeichneten Schlusspunkt erreicht. Dabei ist zunächst einmal bemerkenswert, dass es nicht mehr der Erzähler in seiner Jugend ist, der hier am Grabmal der schönen Verstorbenen steht, sondern ein alt und grau gewordener Efraín, der am Ende des Romans mit unbekanntem Ziel davongaloppiert, ganz jene Weissagung von Carlos erfüllend, er werde wohl den Rest seiner Jahre am Grabe der unvergleichlichen María zubringen. Dies ist kein Wunder: Die Ebenen von Erzählzeit und erzählter Zeit sind zusammengefallen, ein in der Ich-Erzählsituation recht

9 Isaacs, Jorge: María, S. 320.

10 Ebda., Kapitel 63.

11 Ebda., S. 344. 
häufiges Element, das wir schon mehrfach beobachten konnten und das stets den Tod, zumindest den Tod der Geschichte(n), auf romantechnischer Ebene zum literarischen Ausdruck zu bringen versucht.

Aufschlussreich ist aber auch die Tatsache, dass dieses Davongaloppieren, dieses Verschwinden am Horizont mit unbekanntem Ziele, im hispanoamerikanischen Roman mit einer nicht geringen Häufigkeit erscheint. Die Zukunft, so könnten wir diese parallele Gestaltung von Romanschlüssen deuten, ist innerhalb dieser nationalen Allegoresen offen, allerdings oft nicht in einem hoffnungsfrohen, optimistischen, sondern eher in einem dunklen, unbestimmten Sinne. Die Nationbildungsprozesse, welche diese Romane wesentlich thematisieren und vorführen, scheitern fast durchweg und lassen der Zukunft nur eine beschränkte Chance. Das 19. Jahrhundert ist über lange Phasen hinweg für eine große Anzahl der noch jungen lateinamerikanischen Nationen ein verlorenes, ja verschenktes Jahrhundert, das viel aussagt über das Scheitern der an die Macht gekommenen Eliten. Die Helden verschwinden am dunklen Horizont, Die Zeit nach der Kolonie, die postkoloniale Zeit, beginnt im Modus ständiger Irrungen und Wirrungen. Man könnte einen derartigen Romanschluss - wäre er nicht ein literarisches Versatzstück der Romantik - in dieser allegorischen Sichtweise als geradezu prophetisch bezeichnen, denn die Zukunft Lateinamerikas bleibt ungewiss.

Nun aber müssen wir uns gerade an dieser Stelle ein weiteres Mal mit der Verbindung von Erotik und Tod auseinandersetzen, die in diesem kolumbianischen Roman, aber auch schon in seinen französischen Vorbildern und Bezugstexten, eine ganz entscheidende Rolle spielt. Ein kleiner vergleichender Ausflug sei mir hier gestattet: In Eugenio María de Hostos’ La Peregrinación de Bayoán wird die Körperlichkeit der Heldin just in jenem Augenblick zu einem Thema, ja zu einer Obsession des Protagonisten und des Romans selbst, als diese Heldin vom physischen Tod bedroht ist und ihrem Totenbette nahe scheint, oder schon in diesem liegt. Es ist genau jener Augenblick, in welchem der ersehnte Körper der Frau unmittelbar nahe, zugleich aber unerreichbar ist, der durch diese doppelt lesbare Dimension semantisch ungeheuer aufgeladen wird.

Bereits in Chateaubriands Atala war ein weiterer Aspekt miteingearbeitet: der des Inzests. Das Inzesttabu schwebte bereits deutlich über den fast brüderlich-schwesterlichen Beziehungen zwischen Atala und ihrem Chactas, ein literarisches Motiv, das sich selbstverständlich auch in vielen anderen Romanen der Romantik, auch in dem für Isaacs wichtigen Text von Byrons Manfred findet. Es liegt ebenso unverkennbar über Villaverdes Cecilia Valdés, wobei es in diesem Roman freilich - und dies ist selten - insofern gebrochen wurde, als der eheliche 
Sohn und die uneheliche Tochter des alten Gamboa tatsächlich vor ihrem Tod miteinander geschlechtlich und lustvoll verkehren und es sogar zur Geburt einer süßen Frucht dieses Inzests, einer kleinen Mulattin, kommt.

Auch der Roman Sab von Gertrudis Gómez de Avellaneda deutete, wenn auch nur sehr vage, ein Inzesttabu an, da auch Sab ein uneheliches Kind ist, hier allerdings lediglich des Bruders des Vaters seiner Angebeteten. In La Peregrinación de Bayoán erscheint ebenfalls ein Hauch des Inzests in der Geschichte, ist doch die Mutter der so empfindsamen und schwächlich-schönen Angebeteten eine Figur, die für den Protagonisten von großer Wichtigkeit und Ausstrahlungskraft ist, gerade deshalb aber nie beim Namen genannt wird und nur als madre (das heißt als Mutter der Geliebten) oder als mujer (und damit als Ehefrau eines Anderen) erscheint. Ihre Namenslosigkeit verweist auf die inzestuöse Bedrohung des jungen Mannes, der noch immer einer Mutterfigur, einer Figuration seiner eigenen Mutter, anhängt.

Steven Boldy hat in seiner lesenswerten Studie von Jorge Isaacs’ María nachgewiesen, wie sehr auch dieser Roman von inzestuösen Strukturen durchzogen ist. $^{12}$ Wenn Boldy auch die intertextuelle Dimension des Inzestuösen als literarische Konvention vernachlässigt und damit die transgressive Dimension des Texts nach meinem Dafürhalten überbetont, ist es ihm doch gelungen, die vielfältigen inzestuösen Netze und Beziehungen herauszuarbeiten. Nicht allein Marías Herkunft selbst - sie ist die Tochter der Schwester von Efraíns Vater -, sondern mehr noch ihre Überlagerungen mit Mutter und Schwester ihres Geliebten machen sie zu einem Mittelpunkt eines ganzen Bündels inzestuöser und daher verdrängter Liebeswünsche.

Bereits zu Beginn des Romans verbinden sich für den kleinen Efraín die Funktionen von Mutter, Schwester und Geliebter in der kleinen María, deren Bild sachte mit jenem ersterer verschmilzt. Und noch am Ende des Romans schließt der aus Europa herbeieilende junge Mann nicht die bereits verstorbene María, sondern - sagen wir versehentlich - seine eigene Schwester Emma in die Arme, die er mit Küssen überschüttet, ohne dass uns von größerem Widerstand der so Behandelten berichtet würde. Vergessen wir darüber nicht, dass die junge Emma in der Schlüsselszene der gemeinsamen Lektüre von Atala psychoanalytisch verwertbar ihren Arm auf den Arm ihres Bruders gestützt hatte - so unschuldig ist die bruder-schwesterliche Beziehung mithin nicht!

12 Vgl. Boldy, Steven: Jorge Isaacs’s „María“: a Reappraisal. In (ders., Hg.): Before the Boom. Latin American Narrative in the Nineteenth Century. Liverpool: Center for Latin American Studies 1989, S. 40-60. 
Efraín kommt auf seiner übereilten Rückreise aus England im Übrigen zu spät, denn María ist bereits begraben - ein Aspekt, der auch auf die inzestuöse Beziehung zwischen María und Efraíns Vater und die Rivalität zwischen Vater und Sohn aufmerksam macht, muss doch schließlich auch der Vater einräumen, dass er den Tod Marías durch sein Handeln zumindest beschleunigt habe und damit Schuld an ihrem verfrühten Dahinscheiden trägt. Da der Körper der Geliebten also nicht mehr auf dem Totenbett, sondern bereits in der Erde liegt, muss die Szene am Bett der Verstorbenen - und damit die Verbindung von Tod und Erotik, von Eros und Thanatos - nachgeholt werden, was denn auch geschieht.

Denn Efraín spielt mit den Zöpfen seiner María - eine Art Ersatz, der zugleich auf die erotische Dimension von Haaren überhaupt hinweist ${ }^{13}-$, als er im Bett der Geliebten liegt und nun träumt, er habe noch einmal seine María in Armen, eine Situation, zu der es zuvor noch nicht gekommen war. Gewiss: Alles erscheint als ein Traum, ein Alptraum, aus dem der junge Mann mit einem Schrei wieder aufwacht. Doch zeigt sich auch in dieser Passage, dass gerade der Tod des geliebten Wesens Phantasien der Verfügungsgewalt über dessen Körper beflügelt, die im Leben offenkundig erfolgreich unterdrückt worden waren.

Wir dürfen uns an diesem Punkt durchaus fragen, aus welchen Gründen denn die Inzestphantasien gerade im romantischen Roman Hispanoamerikas eine derart präponderante Rolle spielen. Natürlich wären als Gründe zum einen die schon in Atala angelegte literarische Tradition zu nennen sowie - in einem engeren Sinne - die Häufigkeit dieses Motivs innerhalb des französischen Feuilletonromans, der ja für den Aufstieg des Romans auch in Hispanoamerika eine wichtige, vielleicht sogar entscheidende Rolle spielte. Damit hätten wir gleichsam einen innerästhetischen und einen distributionsspezifischen Grund gefunden, wobei letzterer mit dem Inzestmotiv die Notwendigkeit von Spannungserzeugung und -aufrechterhaltung im Feuilleton verbindet.

Doch dies allein scheint mir nicht zu genügen! Das Inzesttabu ist ein generelles und unüberwindliches Tabu, eine Schranke, die gleichwohl von Menschen gesetzt ist und daher die Unüberwindlichkeit mit der vom Menschen beziehungsweise gesellschaftlich gesetzten Konvention verbindet. Es bindet dadurch die Unerreichbarkeit des romantischen Begehrens an sich, da das geliebte, ersehnte Ziel zugleich als nah und doch unerreichbar erscheint, wobei die Grenze eben letztlich nicht von der Natur, sondern von den Gesetzen gesellschaftlichen Zusammenlebens errichtet worden ist. In dieser Ambivalenz sozialer Konvivenz dient es der Inszenierung romantischen Begehrens bezie-

13 Vgl. hierzu Futoransky, Luisa: Pelos. Madrid: Ediciones Temas de Hoy 1990. 
hungsweise Scheiterns und wird zugleich eingebunden in die Funktion einer nationalen Allegorese, deren Misslingen sozusagen en famille und im kleinen Kreise vorgeführt wird.

Damit haben wir einen weiteren wichtigen Grund für die Häufigkeit des Motivs nicht allein in der Romantik, sondern gerade auch in der Romantik Hispanoamerikas dingfest gemacht. Denn es ist die Fusion (verschoben auf die Ebene der unmöglich gemachten körperlich-sexuellen Verbindung von Individuen), die hier nicht durchgeführt werden kann, jene Vereinigung, die von den verschiedenen Nationalitätskonzepten des 19. Jahrhunderts vorausgesetzt und eingeklagt wurde. Die gescheiterte Fusion wird damit in gewisser Weise nicht nur begründet, sondern auch entlastet: Sie ist letztlich unmöglich, da ihr ein Begehren innewohnt, das nicht statthaft ist und zu keiner gesellschaftlich akzeptierten Konvivenz führen kann.

Damit aber verdichtet dieses Motiv in überaus polysemer Weise die Problematik einer gesellschaftlichen Entwicklung der lateinamerikanischen Republiken zu einer tabuisierten Grundfigur, deren Tiefe wir mit Hilfe der hier skizzierten vier Aspekte zwar erkannt, aber längst noch nicht vollständig ausgelotet haben. Deutlich jedoch sehen wir, dass das Motiv in Hispanoamerika zusätzliche gesellschaftliche Funktionen übernimmt, die im französischen Feuilletonroman selbstverständlich nicht vorgesehen sein können und ein bezeichnendes Licht auf jene sozialen Aporien werfen, welche eine gelingende Konvivenz in den jungen Staaten Lateinamerikas unmöglich machten.

Wir stellen auch auf dieser Ebene fest, dass Jorge Isaacs’ Roman María von 1867 in gewisser Weise eine ganze Vielzahl charakteristischer Inhalts- und Textelemente zusammenführt und daher als einer der sicherlich herausragenden Romane nicht nur des kolumbianischen, sondern auch des hispanoamerikanischen neunzehnten Jahrhunderts gesehen werden darf. Seine zentrale Stellung innerhalb einer hispanoamerikanischen Romangeschichte könnte im Übrigen auch damit begründet werden, dass die Romandiegese sozusagen a caballo ist, sich also sowohl auf den südamerikanischen Kontinent, die Karibik (unter Einschluss Jamaicas und der Südstaaten der USA), Mittelamerika, aber auch den Andenraum hin öffnet und zugleich ein Stückchen Europa vermittelt, insofern der London-Aufenthalt des Protagonisten eingeblendet wird.

Sicherlich könnte man an dieser Stelle einfügen, dass die bislang eher vernachlässigte Dimension des Romans jene der gesellschaftlichen Realität ist, die er zumindest auf den ersten Blick sorgfältig ausblendet. Zur Betonung dieser politischen Dimension könnten wir darauf verweisen, dass Jorge Isaac selbst nicht nur Protagonist der Veränderungen auf politischer Ebene war - er wechselte von den Konservativen zu den Liberalen, war zeitweilig Präsident des 
Abgeordnetenhauses und wurde als solcher auch in einem Amtsenthebungsverfahren ausgehebelt, da er zur Insurrektion aufgerufen hatte -, sondern dass er auch als Erbe zweier zweitrangiger Haciendas die Modernisierung und ihre wirtschaftlichen wie gesellschaftlichen Zwänge gerade im Cauca-Gebiet miterlebte.

Denn er verlor nicht nur rasch beide Besitzungen, sondern musste mitansehen, wie diese später von einem US-amerikanischen Einwanderer aufgekauft und wieder in gewinnabwerfende Betriebe umgewandelt wurden. ${ }^{14}$ Doch sollten wir bei alledem nicht vergessen, dass es Isaacs weitaus weniger als etwa José Mármol darum ging, einen politischen oder historischen Roman zu schreiben und dabei die eigene Situierung innerhalb der zeitgenössischen Politik seines Landes zu bestimmen. Dies bedeutet freilich nicht, dass sich sein Roman nicht einer historisch-soziologischen Untersuchung öffnen würde.

Doch Jorge Isaacs schrieb sich nicht in derartige Traditionslinien, sondern weitaus mehr in jene der novela sentimental ein, die - wie wir mehrfach beobachten konnten - in ihrer Andersartigkeit und Unterschiedlichkeit doch nur die Kehrseite jener Münze ist, auf die der Roman auch eines José Mármol setzte. Es handelt sich also nicht um einen politischen, wohl aber um einen politisch deutbaren Liebes- und Lektüreroman. Wir lesen folglich keinen historischen, wohl aber einen Geschichte in Handlungs- und Diegesestrukturen umarbeitenden Roman, in dem vor allem immer wieder die Anverwandlungsformen von Literatur und unterschiedliche Leserfiguren Gestalt annehmen und vorgeführt werden.

Im Zentrum von Jorge Isaacs’ María, so ließe sich aus Sicht unserer Vorlesung und der damit gewählten Perspektive sagen, steht die Verbindung von Lieben, Lesen und Leben, steht die kreative Aneignung von Liebe durch das Lesen, von Lesen durch die Liebe und eine Aneignung von Leseprozessen, die auf das Leben, dessen Haltungen und Handlungen einen gestaltenden und bestimmenden Einfluss nehmen. Das Lesen wird zu einer self-fulfilling prophecy des eigenen Lebens und Liebens, sind die Protagonisten doch letztlich dazu verurteilt, das leben zu müssen, was ihren Helden widerfuhr: So wird María wie Atala sterben und Efraín bis zum Ende seiner Tage das Grab Atalas mit seinen Tränen - den Tränen eines getreuen Lesers - benetzen. Lesen ist gefährlich! Dass ein derartiger Roman in seiner Herausarbeitung einer als überzeitlich entwickelten Liebesgeschichte als Seelendrama nationalpolitisch wirken konnte und wirken sollte, haben wir

14 Vgl. zu den politischen Implikationen Bremer, Thomas: Jorge Isaacs: „María“. In: Roloff, Volker / Wentzlaff-Eggebert, Harald (Hg.): Der hispanoamerikanische Roman. Bd. 1: Von den Anfängen bis Carpentier. Darmstadt: Wissenschaftliche Buchgesellschaft 1992, S. 64-77. 
anhand anderer Beispiele schon zur Genüge beobachten können. María bietet folglich nicht allein die Lektüre einer individuellen Liebesgeschichte, sondern führt uns tief in die gesellschaftlichen (Liebes-)Verhältnisse in den jungen Nationalstaaten Lateinamerikas. Die Liebesgefühle, so scheint es zumindest für die gesellschaftliche Elite zu sein, werden direkt aus Europa importiert. Liebe ist eine erlesene Praxis ... 